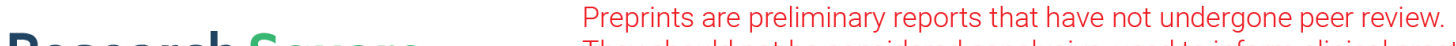 They should not be considered conclusive, used to inform clinical practice, or referenced by the media as validated information. \\ Quantifying nanodiamonds biodistribution in whole cells with correlative iono-nanoscopy
}

\section{Zhaohong Mi}

National University of Singapore

\section{Hong Qi Tan}

National University of Singapore https://orcid.org/0000-0001-7878-4544

\section{Ce-Belle Chen}

National University of Singapore

\section{Yanxin Dou}

National University of Singapore

\section{Chengyuan Yang}

National University of Singapore

\section{Shuvan Prashant Turaga}

National University of Singapore https://orcid.org/0000-0003-1457-8439

\section{Frank Watt}

Centre for lon Beam Applications, NUS

Andrew Bettiol ( $\nabla$ a.bettiol@nus.edu.sg )

National University of Singapore

\section{Article}

Keywords: nanodiamonds biodistribution, correlative iono-nanoscopy, whole-cell correlative imaging

Posted Date: September 4th, 2020

DOI: https://doi.org/10.21203/rs.3.rs-54546/v1

License: (c) (1) This work is licensed under a Creative Commons Attribution 4.0 International License. Read Full License

Version of Record: A version of this preprint was published at Nature Communications on August 2nd, 2021. See the published version at https://doi.org/10.1038/s41467-021-25004-9. 


\title{
Quantifying nanodiamonds biodistribution in whole cells with correlative iono-nanoscopy
}

\author{
Zhaohong Mi', Hong Qi Tann ${ }^{1,3}$, Ce-Belle Chen', Yanxin Dou', Chengyuan Yang', Shuvan Prashant Turaga', \\ Frank Watt ${ }^{1 凶}$, and Andrew A. Bettiol ${ }^{1,2} \bowtie$
}

Correlative imaging and quantification of intracellular nanoparticles with the underlying ultrastructure is crucial for understanding cell-nanoparticle interactions in biological research. However, correlative nanoscale imaging of whole cells still remains a daunting challenge. Here, we report a straightforward nanoscopic approach for whole-cell correlative imaging, by simultaneous ionoluminescence and ultrastructure mapping implemented with a highly focused beam of alpha particles. We demonstrate that fluorescent nanodiamonds exhibit fast, ultrabright and stable emission upon excitation by alpha particles. Thus, by using fluorescent nanodiamonds as imaging probes, our approach enables quantification and correlative localization of single nanodiamonds within a whole cell at sub-40 nm resolution. As an application example, we show that our approach, together with Monte Carlo simulations, can be employed to provide unique insights into the mechanisms of nanodiamond radiosensitization at the single whole-cell level. These findings may benefit clinical studies of radioenhancement effects by nanoparticles in charged-particle cancer therapy. 
$\mathrm{N}$ anoscale imaging of whole cells is essential to observe cellular functions, interactions and dynamics in the native state of the cells. To this end, super-resolution fluorescence techniques, such as stimulated emission depletion microscopy ${ }^{1}$, have become enabling tools to image whole cells of labeled constituents ${ }^{2-4}$. To link the labeled cellular components with the underlying ultrastructure, the super-resolution optical methods have been combined with electron microscopy to provide correlative light-electron imaging capabilities $^{5-7}$. However, electrons essentially suffer from significant scattering when interacting with biological samples as thick as a whole cell, which in turn compromises the resolution ${ }^{8}$. As a result, correlative super-resolution imaging of whole cells remains difficult.

In contrast to electrons, mega-electron-volt $(\mathrm{MeV})$ ions, such as helium ions, offer a significant advantage of being able to penetrate a whole cell with little broadening of the beam spot ${ }^{8,9}$. In our study, the beam spot size of $\mathrm{MeV}$ helium ions can be focused down to sub-30 $\mathrm{nm}$ (see Supplementary Information). As such, the sub-30 nm spatial resolution can be maintained throughout the cell for whole-cell imaging, in stark contrast with electron microscopy. Moreover, a highly-focused beam of $\mathrm{MeV}$ helium ions allows imaging of cell structure by measuring ion energy loss in the scanning transmissionion mode $\mathrm{e}^{9,10}$, whilst simultaneously performing luminescence imaging by collecting luminescent photons upon excitation by helium ions ${ }^{11}$, at super resolutions. Therefore, we hypothesize that a correlative iono-nanoscopic approach can be implemented for whole-cell luminescence and ultrastructure mapping in a single imaging experiment.

However, $\mathrm{MeV}$ ions can be destructive to conventional fluorescent probes (organic dyes, fluorescent proteins, quantum dots and so on $)^{10,12}$. Recently, fluorescent nanodiamonds, particularly those engineered with nitrogen-vacancy (NV) color centers ${ }^{13}$, have gained increasing attention for use as biomarkers in biological and biomedical imaging ${ }^{14-17}$, and as magnetometers in nanoscale quantum biosensing ${ }^{18-21}$. The increased interest in nanodiamonds can be attributed to their biocompatibility, sustained fluorescence, consistent photostability, and prolonged coherence time of the NV centers $^{22}$. In addition, fluorescent nanodiamonds hosting NV centers can emit in the far-red spectral domain, at a wavelength which is out of the range of most autofluorescent components of cells $\mathrm{s}^{10,23}$. Therefore, nanodiamonds with NV centers could be ideal fluorescent probes for ion beam imaging of cells.

The stimulation of $\mathrm{NV}$ centers has been implemented optically $^{13,24,25}$, electrically ${ }^{26,27}$, and also in the form of cathodoluminescence ${ }^{28,29}$. Here, we report the efficient excitation of $\mathrm{NV}$ centers in nanodiamonds by a focused beam of $\mathrm{MeV}$ helium ions, which results in photon emission in the form of ionoluminescence. By using nanodiamonds as imaging probes, we propose a correlative iono-nanoscopic approach for ionoluminescence and ultrastructure imaging in whole cells.

\section{Results}

Instrumentation and implementation of ionoluminescence nanoscopy. In our approach, a highly-focused beam of helium ions (1.6 $\mathrm{MeV} \alpha$-particles) produced with an ion accelerator was instrumental in generating ionoluminescent photons in nanodiamonds (Fig. 1a). The primary beam spot sizes are defined by a set of objective slits, followed by the beam passing through another set of collimating slits, aiming to limit the beam aberrations. The $\alpha$ - beam is then electrostatically raster-scanned before being demagnified by a magnetic-lens-based focusing system (Supplementary Fig. 1 and Note 1). The beam spot sizes achieved in this work are $22 \mathrm{~nm}$ horizontally and $27 \mathrm{~nm}$ vertically (Supplementary Fig. 2 and Note 2). A sample comprising fluorescent nanodiamonds prepared on a silicon nitride membrane (100-nm thick) was positioned precisely at the focal plane of the $\alpha$-beam. Under excitation by $\alpha$-particles, NV centers in the nanodiamonds (structure shown in Fig. 1b) emit ionoluminescent photons, which are captured by either a photomultiplier tube for ionoluminescence mapping or a spectrometer for spectroscopic characterization. Concurrently, the energy-loss of the transmitted $\alpha$-particles, which is proportional to the areal density of the sample ${ }^{10}$, was measured by a silicon surface barrier detector to generate density maps of the sample (Supplementary Fig. 3).

To demonstrate the capability of ionoluminescence nanoscopy, an image of the nanodiamonds was acquired by recording the ionoluminescent photon counts in a $1024 \times 1024$-pixel array (Fig. 1c), at an $\alpha$-particle count rate of around 12000 per second (beam current $\sim 2 \mathrm{fA}$ ). A representative cross-sectional line profile of two single nanodiamonds was collected and fitted with a modified Gaussian function (Fig. 1e and Supplementary Note 2). This result suggests a sub-40 nm resolution of the ionoluminescence image, which is further confirmed by measuring an area containing two nanodiamonds with a separation distance approximating the resolving limit (Fig. 1f). By comparison, an optical confocal image of the same area of the nanodiamond sample was also acquired (Fig. 1d), and as expected this image exhibits much reduced spatial resolution due to optical diffraction.

Mechanistic study of ionoluminescence in nanodiamonds. We next investigated the mechanistic processes that govern ionoluminescent photon emission upon $\alpha$-particle excitation. We first performed spectroscopic photoluminescence measurement of the nanodiamonds under excitation by a 532-nm laser (Fig. 2a). The nanodiamonds exhibit characteristic emission of sharp zero-phononlines $\left(575 \mathrm{~nm}\right.$ for neutral $\mathrm{NV}^{0}$, and $637 \mathrm{~nm}$ for negatively-charged $\mathrm{NV}^{-}$) and their associated phonon sidebands upon 532-nm excitation, confirming that the nanodiamonds host both $\mathrm{NV}^{0}$ and $\mathrm{NV}^{-}$emitting centers. In contrast, a 405-nm laser can only excite $\mathrm{NV}^{0}$ (Fig. 2b). This is in line with the established fact that high-energy photons (energy above $2.76 \mathrm{eV}$ ) are beyond the absorption band of $\mathrm{NV}^{-}$centers ${ }^{24,25}$. Intriguingly, when $\mathrm{MeV} \alpha$-particles (with $10^{5}$ times the energy of $405-\mathrm{nm}$ photons) are used as excitation sources, both $\mathrm{NV}^{0}$ and $\mathrm{NV}^{-}$ centers are turned on, resulting in characteristic ionoluminescence emission (Fig. 2c). We thus speculate that $\alpha$-particle excitation combines the excitation regimes of both low-energy and high-energy photons.

Figure 2 d,e presents a proposed mechanism to elucidate the origin of ionoluminescence emission in NV centers on $\alpha$-particle excitation. Other than direct energy-level transition with optical photon excitation, the interaction of $\mathrm{MeV} \alpha$-particles with atomic electrons predominantly leads to inelastic energy-loss through an atomic ionization process, resulting in the ejection of secondary electrons (Fig. 2d). We carried out kinetic simulations on the production cross-sections of the ionized secondary electrons using a Hansen-Kocbach-Stolterfoht theoretical model ${ }^{8}$. The calculation result shows that the secondary electrons possess kinetic energies 

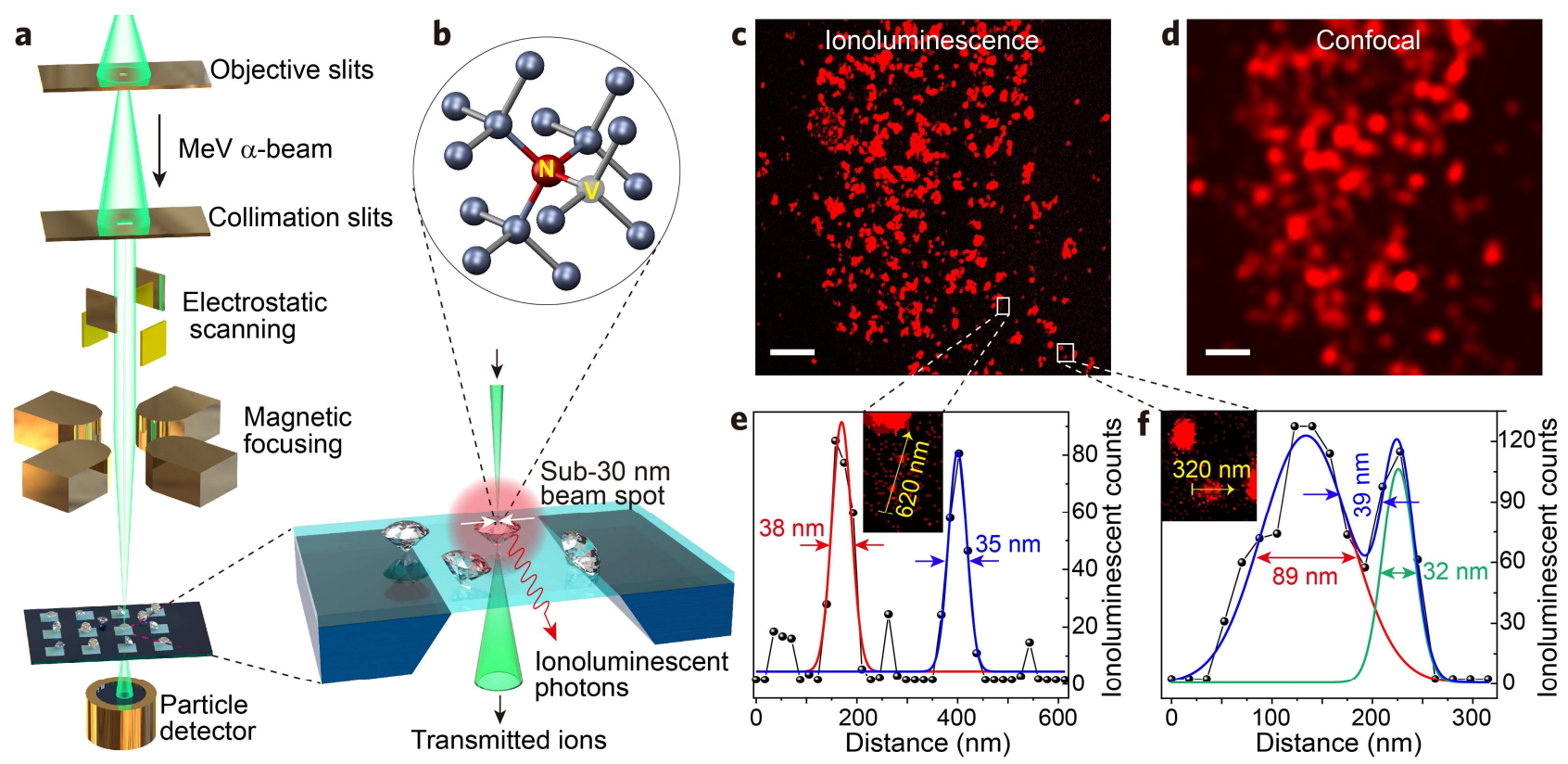

Fig. 1 | Ionoluminescence nanoscopy. a, Schematic illustration of the basic beam optics and experimental setup. Note that ionoluminescent photons emitted from the nanodiamonds induced by the $\alpha$-beam are collected with a parabolic-mirror based system and detected with a photomultiplier tube for ionoluminescence mapping (see Supplementary Fig. 3). b, Nitrogen-vacancy (NV) center in the diamond crystalline structure. c, lonoluminescence image of the nanodiamonds through $\alpha$-particle excitation. Scale bar, $2 \mu \mathrm{m}$. d, Confocal image of the same region of the nanodiamond sample taken by using 543 -nm laser excitation. Scale bar, $2 \mu \mathrm{m}$. e, Cross-sectional line profile extracted along the arrow shown in the inset of a high-magnification image that corresponds to the region of interest marked in c, demonstrating the discrimination of two single nanodiamonds with a size of $38 \mathrm{~nm}$ and $35 \mathrm{~nm}$, respectively. $\mathbf{f}$, Cross-sectional line profile extracted along the arrow depicted in the inset of a high-magnification image that hosts two single nanodiamonds with a separation distance approximating the resolving limit, directly indicating a sub-40 $\mathrm{nm}$ resolution of the ionoluminescence image.

which range from $0.1 \mathrm{eV}$ up to about $1000 \mathrm{eV}$ (Fig. 2d). Of the energetic secondary electrons, they electronically transport in the diamond lattice, leaving holes in the valance band. The hot electrons and holes are then rapidly thermalized to the edges of their corresponding occupying bands (Fig. 2e). These electron-hole pairs, carrying kinetic energies that match the absorption bands of $\mathrm{NV}^{0}$ or $\mathrm{NV}^{-}$, may travel up to the $\mathrm{NV}$ emission centers, where indirect carrier recombination through a Shockley-Read-Hall process will dominate ${ }^{27}$. As a result, both $\mathrm{NV}^{0}$ and $\mathrm{NV}^{-}$centers emit characteristically through this NV-defect-assisted recombination process.

It should be noted that a radiative band-to-band recombination process, in which the interband energy release $(\sim 5.47 \mathrm{eV})$ corresponds to a wavelength of $227 \mathrm{~nm}$, could also occur (Fig. 2e). But the released photons are unlikely to excite $\mathrm{NV}^{0}$ and $\mathrm{NV}^{-}$directly since the absorption ranges of $\mathrm{NV}^{0}$ and $\mathrm{NV}^{-}$are approximately $400-575 \mathrm{~nm}$ and $450-637 \mathrm{~nm}$, respectively ${ }^{24}$. Notably, however, the energy released from this radiative recombination is sufficient to ionize the negatively charged defects, thereby converting $\mathrm{NV}^{-}$to $\mathrm{NV}^{0}$, similar to that of far-UV excitation ${ }^{30}$. And this could be evidenced by the weakened emission of $\mathrm{NV}^{-}$centers whose phonon sidebands are suppressed under $\alpha$-particle excitation, compared with the distinct phonon sidebands of $\mathrm{NV}^{0}$ centers (Fig. 2c).

We took a further step and measured the ionoluminescence properties of the nanodiamonds. Upon excitation with a pulsed beam of $\alpha$-particles, these nanodiamonds exhibit a very fast response with an ionoluminescence decay time of $28.5 \mathrm{~ns}$ (Fig. 2f and
Supplementary Fig. 4). The fast response makes it possible to avoid overlapped counting of photons when performing fast ionoluminescence imaging. Also, the nanodiamonds present a superior performance regarding the relative ionoluminescence yield, as compared with lanthanide-doped nanocrystals ${ }^{11}$, semiconducting quantum dots, and organic dyes that are typically employed for bioimaging (Fig. 2g). Moreover, we observed a pronounced resistance of these nanodiamonds to iono-bleaching (Fig. 2h), a phenomenon associated with the reduction in emission intensity with accumulated ion fluence. The remarkable brightness and iono-stability suggest that nanodiamonds are ideally suited for ionoluminescence imaging under harsh $\alpha$-particle irradiation.

Correlative and quantitative iono-nanoscopy of whole cells. The mechanistic understanding of ionoluminescence facilitates our subsequent investigations into whole-cell correlative imaging of nanodiamonds by using a focused $\alpha$-beam. As a proof-of-concept demonstration, HeLa cells incubated with the nanodiamonds were grown onto a 100-nm-thick silicon nitride membrane. A cell of interest was then concurrently imaged in ionoluminescence mode and transmission-ion mode sorted with dual digital channels, by recording the counts of ionoluminescent photons and energy-loss of those transmitted $\alpha$-particles pixel by pixel, respectively. As a result, a luminescence map that shows the biodistribution of the nanodiamonds and a density map that illustrates cellular structures can be simultaneously obtained (Fig. 3a and Supplementary Fig. 8). This approach thereby robustly validates whole-cell correlative 


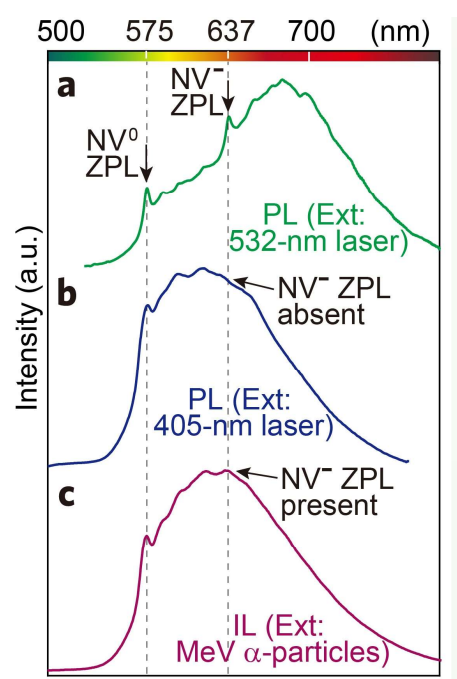

2.1561 .945 d

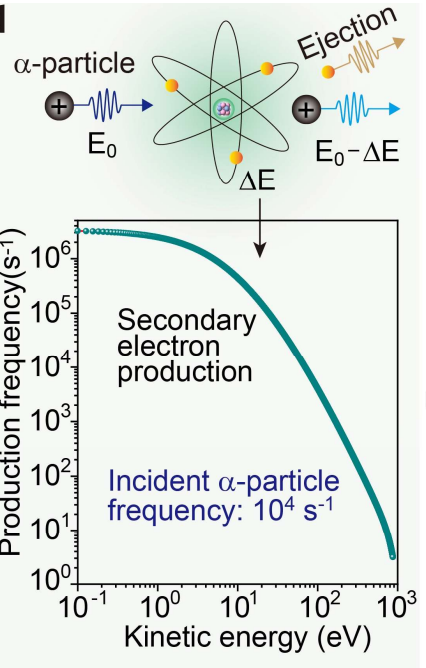

Ionization radiation e Secondary electron

Thermalization Conduction band

$\zeta$ Electron of

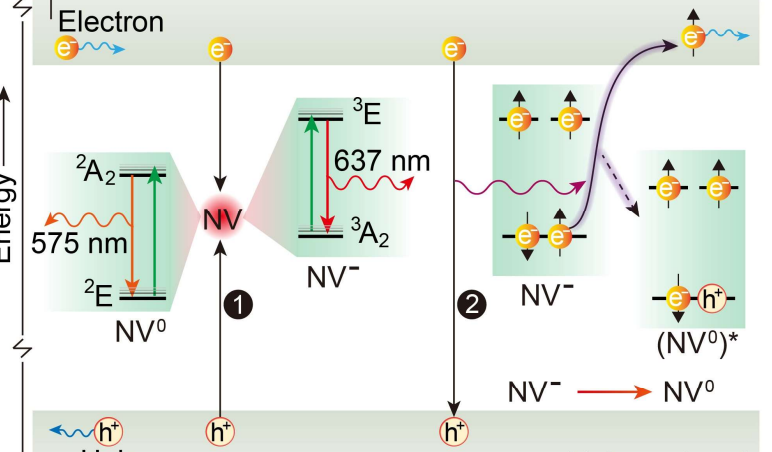

Valance band

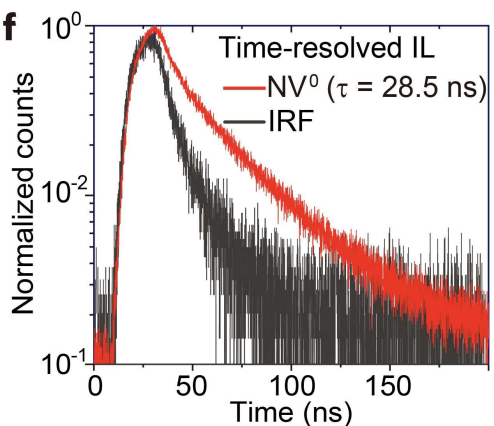

\begin{tabular}{|c|c|}
\hline Perovskite QDs & 1.00 \\
\hline Nanodiamonds & 0.88 \\
\hline \multicolumn{2}{|c|}{ Upconversion nanocrystals 0.67} \\
\hline CdSe/ZnS QDs & 0.28 \\
\hline Fluorescein dyes & 0.18 \\
\hline
\end{tabular}

Ionoluminescence (IL)

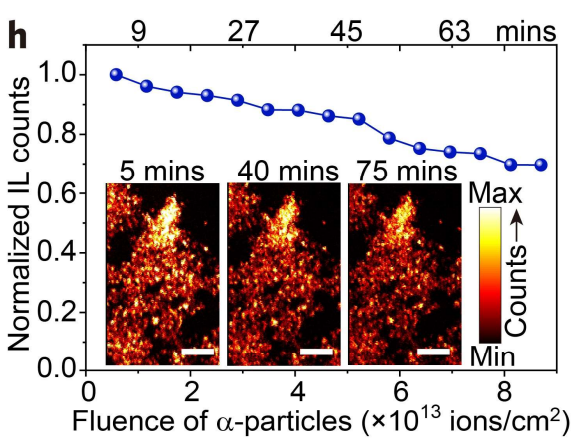

Fig. 2 | Mechanistic investigation and characterization of ionoluminescence in nanodiamonds. a, Photoluminescence (PL) spectrum of nanodiamonds excited with a 532-nm laser. b. Photoluminescence spectrum of nanodiamonds excited with a 405-nm laser. c, lonoluminescence (IL) spectrum of nanodiamonds excited with a beam of $1.6 \mathrm{MeV} \alpha$-particles. $\mathbf{d}$, Illustration of $\alpha$-particle-induced atomic ionization in producing secondary electrons (top), through energy deposition ( $\Delta \mathrm{E}$ ) of the bombarding $\alpha$-particles (energy of $\mathrm{E}_{0}$ ), and calculated energy distribution of the secondary electrons (bottom) in a nanodiamond. e, Proposed mechanism of ionoluminescence through $\alpha$-particle excitation. Process 1 represents NV-defect-assisted recombination which results in the emission of $\mathrm{NV}^{0}$ and $\mathrm{NV}^{-}$. Process 2 represents interband recombination which results in the conversion of $\mathrm{NV}^{-}$to $\mathrm{NV}^{0}$ through ground-state ionization of $\mathrm{NV}^{-}$, forming $\mathrm{NV}^{0}$ in its excited state of $\left(\mathrm{NV}^{0}\right)^{\star} . \mathbf{f}$, Time-resolved ionoluminescence measurement. Note that the instrumental response function (IRF) was determined by measuring the ionoluminescence response of a fast-decay material (Supplementary Fig. 5 and Note 3). g, Relative ionoluminescence yield measurement. Note that the ionoluminescence yields of nanodiamonds, upconversion nanocrystals $\left(\mathrm{NaYF}_{4}: \mathrm{Yb} / \mathrm{Tm}\right), \mathrm{CdSe} / \mathrm{ZnS}$ quantum dots (QDs), and fluorescein dyes (FITC-1907) were normalized with a perovskite-QDs scintillator (Supplementary Figs. 6, 7 and Note 4). h, lonoluminescence intensity profile as a function of the accumulated fluence of $\alpha$-particles, showing a considerable iono-bleaching resistance of the nanodiamonds. The inserted images, taken at different time intervals (5, 40, and 75 mins), indicate that the emission brightness of the nanodiamonds remains essentially unaltered over time. Scale bars are $2 \mu \mathrm{m}$.

localization of intracellular nanodiamonds when the ionoluminescnece map and the density map are overlaid (Fig. 3b). More importantly, the single-particle discrimination capability of ionoluminescence nanoscopy, together with the development of a statistical counting method based on Poisson distribution (see Methods), enables quantitative imaging of single nanodiamonds within the cell. For illustration, we have been able to measure the number of single nanodiamonds in terms of their distance to the nuclear boundary within the whole cell (Fig. 3c). Furthermore, a total of 11 cells were measured to chart the distribution of nanodiamondsto-nucleus distance (Fig. 4a and Supplementary Fig. 9).

Mechanistic investigation of nanodiamond radiosensitization effects. The capability of our approach in achieving whole-cell quantitative localization of nanodiamonds could pave the way to new research areas. For instance, nanodiamonds have been demonstrated in vitro as radiosensitizers to enhance the effect of cancer radiotherapy ${ }^{31}$, but clear pathways that might be responsible for the effect at the subcellular level are still in dispute. The cell nucleus has been considered as the main target for ionizing radiation, and nanoparticles generally do not enter the nucleus (evidenced in Fig. 4a). Such assumptions imply that any radio-enhancement effect is derived from the secondary electrons that emanate from the nanoparticles located in the cytoplasm when interacting with primary radiation. The travel ranges of the secondary electrons and subsequent reaction products can be simulated. However, to quantitatively assess if these secondary products have a range that can affect nuclear DNA or just be able to damage the surrounding cytoplasmic components, we need to know the nanoparticle-nucleus distance in a whole cell. This is now achievable by using our 

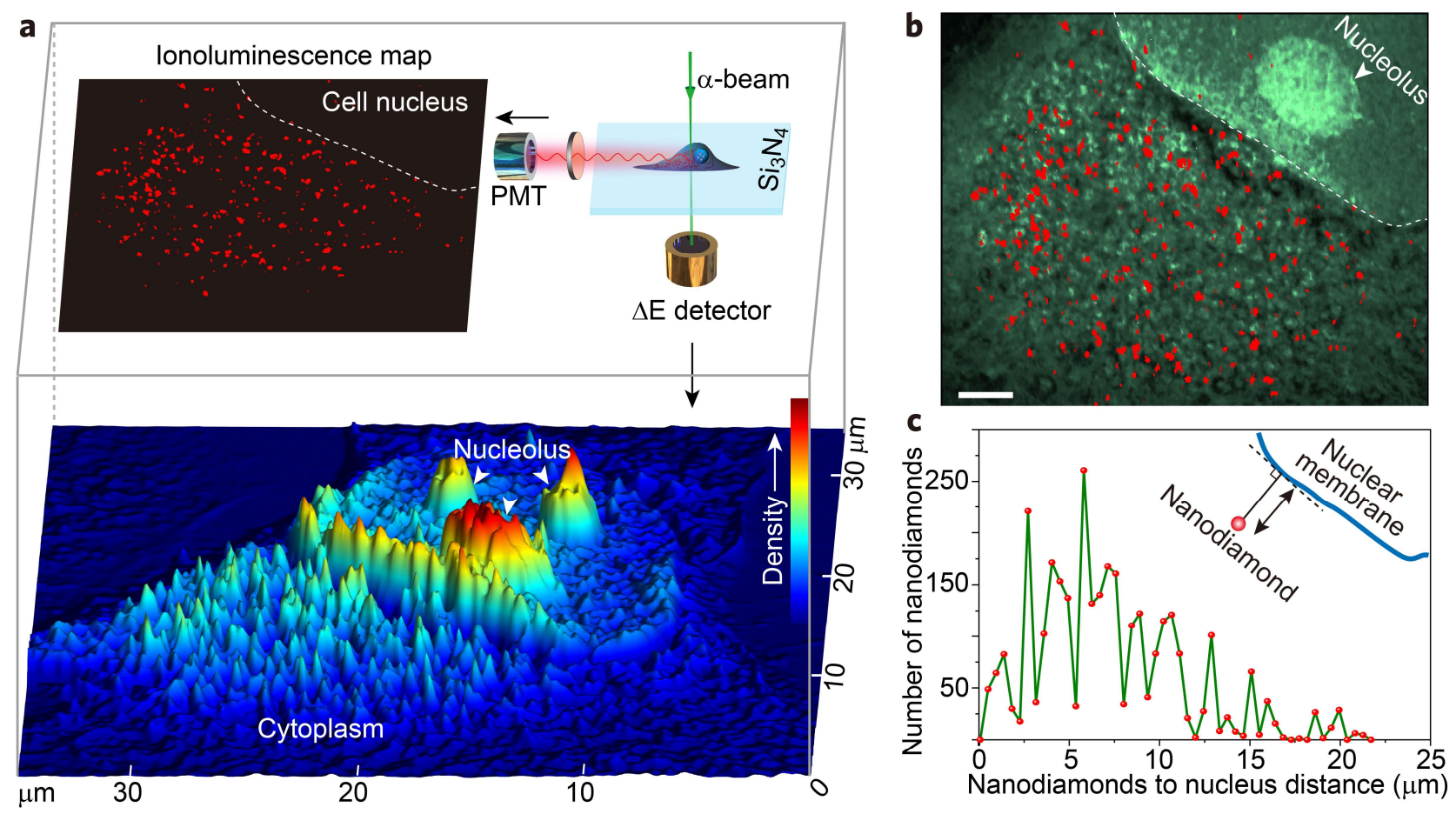

Fig. 3 | Correlative ultrastructure and ionoluminescence mapping towards quantitative localization of single nanodiamonds in a whole HeLa cell. a Demonstration of correlative ultrastructure and ionoluminescence imaging of the HeLa cell with endocytosed nanodiamonds, realized in a single imaging experiment. The basic experimental design (top right) enables simultaneous acquisition of luminescence image of the nanodiamonds and 3D rendering of cell ultrastructure, by capturing $\alpha$-particle-induced photons with a photomultiplier tube (PMT) and by detecting the energy-loss ( $\Delta \mathrm{E}$ ) of the transmitted $\alpha$-particles through a Si surface barrier detector, respectively. $\mathbf{b}$, Overlay of the structural and luminescent images presented in $\mathbf{a}$, showing localization of nanodiamonds in the cell. Scale bar, $2 \mu \mathrm{m}$. c, Measurement of the number of intracellular nanodiamonds displayed in $\mathbf{b}$ in terms of their distance to the cell nuclear boundary.

correlative iono-nanoscopic approach. In particular, a minimum distance of $354 \mathrm{~nm}$ between the closest nanodiamonds and the nucleus was determined from our study (Fig. 4a).

As an example, we consider here the case of proton irradiation in cancer therapy ${ }^{32}$, and assess the validity of nanodiamonds-mediated radiosensitization mechanisms by using Geant4-DNA Monte Carlo simulations (Fig. 4b). In our model, we assume the nanodiamond is spherical with a diameter of $100 \mathrm{~nm}$. The energy spectrum of protoninduced secondary electrons escaping the nanodiamond at its surface was calculated (Fig. 4c). These secondary electrons were assumed to serve as a source to produce reactive oxygen species when they interact with the cell cytoplasm (simulated with water). Monte Carlo simulations using Geant4-DNA were performed to trace secondary electrons and reactive radicals (the most harmful hydroxyl radical as an example), and their end-of-ranges were recorded (Figs. 4d, e).

Next, we considered the secondary electrons and hydroxyl radicals that could travel more than $354 \mathrm{~nm}$ to reach the cell nucleus (Fig. 4f). To deliver a typical clinically-relevant dose of $4 \mathrm{~Gy}$ in a HeLa cell, a nanodiamond will experience the impact by 0.1 protons on average (Supplementary Note 5). By integrating the total counts from Fig. 4f, the secondary electrons and the hydroxyl radicals that can reach the nucleus were determined to be 0.003 and 0.162 per 0.1 proton impact respectively, which is extremely low. Moreover, hydroxyl radicals react rapidly and are unable to directly penetrate the nuclear membrane ${ }^{33}$. These results quantitatively indicate that neither secondary electrons nor hydroxyl radicals derived from the nanodiamond can directly damage nuclear DNA. Since the nanoparticle radiosensitization effect for proton irradiation has been experimentally established ${ }^{34,35}$, we speculate that other mechanisms should be responsible for the effect. As expected, our simulation suggests that hydroxyl radicals are highly localized near the nanodiamond surface (Fig. 4e). Therefore, the primary cellular targets for radiosensitization could be organelles, their lipid membranes or cytosolic proteins in close proximity to the nanodiamonds. These cellular components may mediate biological responses of radiosensitization ${ }^{36,37}$.

\section{Discussion}

We demonstrated correlative iono-nanoscopy as a powerful technique to image, localize and quantify fluorescent nanodiamonds at sub-40 $\mathrm{nm}$ resolution throughout a whole cell, by taking advantage of a highly focused $\mathrm{MeV} \alpha$-beam. Our findings reveal fast, ultrabright and stable ionoluminescence emission of fluorescent nanodiamonds on excitation with $\mathrm{MeV} \alpha$-particles. The $\mathrm{NV}^{0}$ emitting centers in nanodiamonds were found to dominate the ionoluminescence emission, primarily through NV-defects-assisted recombination. Furthermore, the experimental results of whole-cell correlative localization of nanodiamonds, together with Geant4-DNA Monte Carlo simulations, enable us to shed light on nanodiamond radiosensitization effects at the sub-cellular level. Our findings indicate that the mechanism of nanodiamond-mediated radiosensitization for proton irradiation does not occur through 

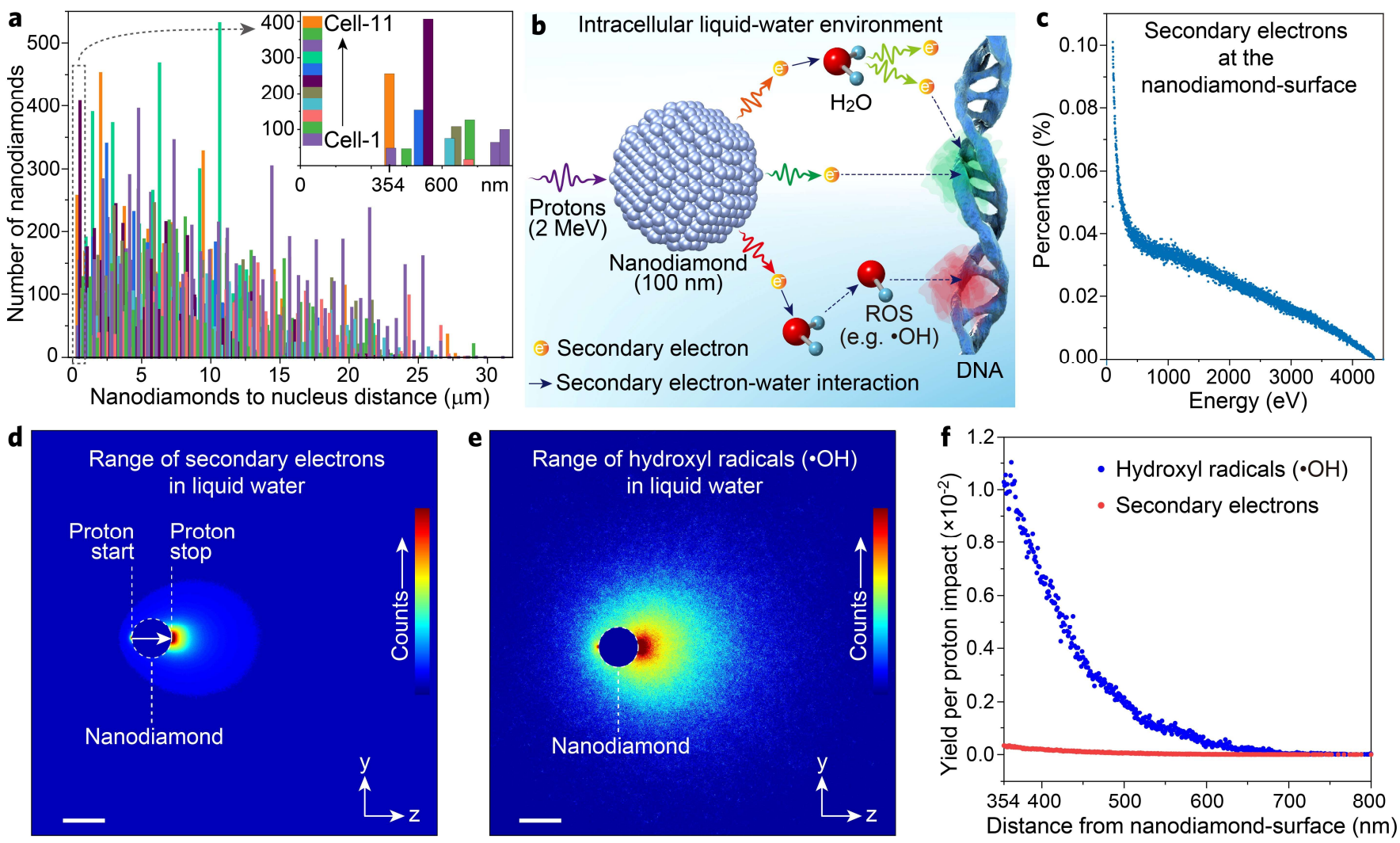

Fig. 4 I Evaluation of nanodiamonds as proton radiosensitizers. a, Histogram showing the distribution of nanodiamond-to-nucleus distance that was experimentally measured within $11 \mathrm{HeLa}$ cells. The magnified inset indicates that zero nanoparticles were found within $354 \mathrm{~nm}$ of the nuclear boundary. $\mathbf{b}$, Plausible mechanisms of nanodiamond-mediated proton radiosensitization in damaging nuclear DNA. Secondary electrons can be induced by the 2-MeV protons when they penetrate a nanodiamond. The secondary electrons that emanate from the nanodiamond, as well as subsequent generations of induced electrons, can either damage nuclear DNA directly, or ionize intracellular water molecules in producing reactive oxygen species (ROS) to react with nuclear DNA indirectly. c, Calculated energy distribution of the secondary electrons escaping the nanodiamond at its surface, simulated with Geant4-DNA. d, Image showing range distributions of the secondary electrons in liquid water, simulated with Geant4-DNA. Note that we assumed the protons travel only within the nanodiamond (100 nm diameter). Scale bar, $100 \mathrm{~nm}$. e, Image showing range distributions of hydroxyl radicals $(\cdot \mathrm{OH})$ in liquid water, simulated with Geant4-DNA. Scale bar, $100 \mathrm{~nm}$. $\mathbf{f}$, Measurement of the number of hydroxyl radicals and secondary electrons per proton impact as a function of their distances from the nanodiamond-surface. Note that only those traveling more than $354 \mathrm{~nm}$ (the minimum nanodiamond-to-nucleus distance determined in a) were taken into account.

damaging nuclear DNA by induced secondary electrons or hydroxyl radicals. Instead, our results suggest that the high yield of localized reactive species derived from nanodiamonds could be responsible for the radio-enhancement effects in proton irradiations.

Though further experimental and clinical studies should be performed to validate our results, we believe our findings can deepen the fundamental understanding of nanoparticle-radiosensitization mechanisms to facilitate charged-particle cancer therapy in the era of precision medicine $e^{32,38}$. In addition, our unique technique of correlative iono-nanoscopy of whole cells may benefit many other emerging applications, for example, intracellular delivery and screening of potential COVID-19 therapeutics and vaccines ${ }^{39,40}$.

\section{Methods}

Nanodiamond sample preparation. The hydroxylated nanodiamonds (900 NV centers per particles) used in this study were sourced from Adámas Nanotechnologies. The nanodiamonds were diluted to $100 \mu \mathrm{g} / \mathrm{ml}$, sonicated for 5 minutes, dropped onto a 100-nm-thick silicon-nitride membrane substrate, and air-dried before being transferred for ionoluminescence nanoscopic imaging and scanning electron microscopic (SEM: JEOL JSM-6700F) analysis. SEM characterizations and size measurement of the nanodiamonds can be found in Supplementary Fig. 10 and Note 6.

Cell sample preparation. Human cervical carcinoma cells (HeLa) were cultured in Dulbecco's Modified Eagle's medium (DMEM) supplemented with fetal bovine serum $(10 \%)$, antibiotics penicillin $(100 \mathrm{units} / \mathrm{ml})$ and streptomycin $(100 \mu \mathrm{g} / \mathrm{ml})$ at $37^{\circ} \mathrm{C}$ in $5 \% \mathrm{CO}_{2}$ atmosphere. HeLa cells were then seeded onto sterilized silicon-nitride-membrane (100 $\mathrm{nm}$ thick) substrates at an estimated density of 10000 cells $/ \mathrm{cm}^{2}$ and allowed to adhere overnight. After attachment, control cells were briefly rinsed with phosphate-buffered saline (PBS) and further incubated with fresh supplemented DMEM for 16 hours. Other cells awaiting nanodiamond uptake were incubated similarly but with the addition of nanodiamonds (100 $\mu \mathrm{g} / \mathrm{ml}$ ) in supplemented DMEM. Cells were then washed thoroughly with PBS prior to fixation in formaldehyde (4\%). Fixed cells were intermediately dehydrated by passage through an increasing ethanol gradient, followed by complete dehydration using critical point drying.

Instrumentation and correlative imaging. Confocal imaging of nanodiamonds was performed by using a laser-scanning microscope (Zeiss LSM 510) equipped with a $543 \mathrm{~nm}$ helium-neon laser. For ionoluminescence analysis, the $\mathrm{MeV} \alpha$ particles were produced with a single-ended electrostatic particle accelerator $\left(\right.$ HVEE Singletron ${ }^{\mathrm{TM}}$ ) inbuilt with a radio-frequency ion source. The 
nanodiamond sample was placed in a vacuum chamber $\left(10^{-6} \mathrm{mbar}\right)$ at a position situated along the $\alpha$-beam path at the beam focus. The $\alpha$-particle-induced photons were collected with a customized double-piece parabolic mirror ${ }^{41}$, and captured either by a photomultiplier tube (Hamamatsu R7400P) for luminescence imaging or by a spectrometer (Ocean Optics QE65000) for spectroscopic characterization. Concurrently, the front and rear openings designed on the parabolic mirror allow the focused $\alpha$-beam to pass through the sample and exit. A Si surface barrier detector (Ortec) was placed downstream to measure energies and the number of transmitted $\alpha$-particles after penetrating a given sample. The energy loss, which is proportionally related to the sample's density at each pixel within the scanned area was thus determined. When the sample is a cell, the photon-counts and energy-loss data were collected and processed using the IONDAQ data acquisition system ${ }^{42}$ to generate correlative ionoluminescence and structure maps of the cell, respectively.

Time-resolved ionoluminescence. The time-resolved ionoluminescence system was constructed by driving a beam blanker with a high-voltage pulse generator (AVTech AV-1010-B, rise-time $10 \mathrm{~ns}$ ) to discretize the $\alpha$-beam, and using the same signal to synchronize with the time-correlated single-photon counting hardware (PicoQuant Timeharp 260 PICO). A hybrid photomultiplier detector (PicoQuant PMA Hybrid 40), coupled with a band-pass filter, was employed to detect single ionoluminescent photons associated with $\mathrm{NV}^{0}$ centers in nanodiamonds. A singlephoton statistical histogram was formed over multiple cycles by registering photon arrivals per time bin (50 ps), referenced by using a fast-decay InGaN quantumwell material ${ }^{43}$. The lifetime of the $\mathrm{NV}^{0}$ centers was thus determined by fitting the histogram with an exponential decay function.

Relative ionoluminescence yield measurement. The ionoluminescence yield of a luminescent nanoparticle is related to many factors, such as the nanoparticle size, light collection and detection efficiency, energy deposition and beam-current fluctuation of the incident $\alpha$-particles. Therefore, it is difficult to determine the absolute ionoluminescence quantum yields of nanoparticles. Instead, we have been able to perform relative ionoluminescence yield measurements. By using the same light collection and detection geometry, we simultaneously took an ionoluminescence image and a scanning transmission ion microscopy ${ }^{10}$ image of each kind of luminescent nanoparticles employed in our study. Thereafter, the number of ionoluminescence counts, the number of incident $\alpha$-particles and their energy-loss at each imaging pixel were acquired from the list-mode data files of these two images. The number of ionoluminescence counts was then divided by the counts and energy-loss of $\alpha$-particles. Finally, a relative ionoluminescence yield was obtained by accumulating the weighted ionoluminescence counts and normalized with a referenced inorganic perovskite quantum-dots scintillator ${ }^{44}$ whose ionoluminescence yield was set to be unitary.

Nanodiamond counting in single whole cells. We proposed a semiautomatic method for statistical nanoparticle counting in ionoluminescence images of a single whole cell, based on using the technique of image segmentation and Poisson statistics. Our approach primarily depends on the identification of single nanodiamonds and their clusters by differentiating them from the background through the correlation between the ionoluminescence image and the correlated structural image, which is demonstrated in Fig. 3. For each pixel of the ionoluminescence image, we consider $N$ ionoluminescent photons are registered by the detector, $N_{p}$ nanodiamonds are present at that pixel, the average number of photons emitted per nanodiamond is $\lambda$, and $\lambda N_{p}$ is thus the average number of photon counts contributed by each particular pixel, which we assumed follows Poisson distribution. On the other hand, since the registered photon counts $N$ is dependent on the number of nanodiamonds present in the corresponding pixel $\left(N_{p}\right)$, a Poisson distribution assumption for $N_{p}$ is also needed to fully capture the statistical property of the photon counts detected. Therefore, a joint probabilitymass-function for $N$ and $N_{p}$ can be given by a double Poisson model which is presented in equation (1):

$$
P(N \mid \lambda, \beta)=\sum_{N_{p}=0}^{\infty} \frac{e^{-\beta}(\beta)^{N_{p}}}{N_{p} !} \frac{e^{-\lambda N_{p}}\left(\lambda N_{p}\right)^{N}}{N !}
$$

where $\beta$ represents the average number of nanodiamonds in each pixel that was recognized as a part of the identified single nanodiamonds and their clusters, and $\lambda \beta$ thus equals to the average number of photons detected per pixel occupied by those single nanodiamonds and clusters. Note that in equation (1), $N$ is the only variable that is experimentally detectable, and the parameters $\lambda$ and $\beta$ are determined by fitting experimental data. If the total number of ionoluminescent counts after background deduction is $C$, the number of pixels containing nanodiamonds is equal to $C /(\lambda \beta)$. Lastly, if each nanodiamond occupies $A$ pixels, the total number of nanodiamonds present in the ionoluminescence image is calculated as $C /(A \lambda \beta)$. In this step, considering that the size uniformity of nanodiamonds is also crucial to the counting accuracy, we expressed $A$ in terms of the size distribution function that is determined in Supplementary Fig. 10. The total number of the namodiamonds in each of the 11 HeLa cells we measured is shown in Supplementary Table 1. Detailed information on image segmentation is provided in Supplementary Note 7 and Supplementary Fig. 11.

Monte Carlo simulations of nanodiamond-mediated radiosensitization. Simulations were performed based on using the Geant4-DNA package which is an extension to the Geant4 (version 10.5) Monte Carlo toolkit ${ }^{45}$. Because the default interaction medium in Geant4-DNA processes is liquid water, in the first step we have chosen G4EmStandardPhysics_option 4 constructor that includes generalpurpose physics models to describe the interaction of protons with the inside of a nanodiamond. This option allows the tracking of secondary electrons with energy down to $100 \mathrm{eV}$. In the simulation, we assumed a $100-\mathrm{nm}$ diameter of the nanodiamond, and $1 \times 10^{7}$ protons with an energy of $2 \mathrm{MeV}$ were used to reach a reasonable statistical accuracy. Thereafter, the secondary electrons that escape the nanodiamond at its surface were filed to source the interaction with liquid water outside the nanodiamond, which was simulated by using the G4EmDNAPhysics_option 4 constructor that is incorporated in the Geant4-DNA package, allowing tracking of induced electrons with energy down to $10 \mathrm{eV}$ in this case. The step size for all induced electrons was set to $5 \mathrm{~nm}$. Meanwhile, the G4EmDNAChemistry constructor was chosen to simulate and track up to 7 chemical species (Supplementary Table 2), within a time domain of $1 \mu \mathrm{s}$. The counts of induced secondary electrons and hydroxyl radicals were sorted based on their stop-sites into two pixel-arrays, respectively, to indicate their ranges in water by coding with MATLAB. Three-dimensional presentations of the travel ranges of the induced secondary electrons and the hydroxyl radicals are provided in Supplementary Fig. 12.

\section{Data availability}

The datasets that support the findings of this study are available from the corresponding authors on reasonable request.

\section{References}

1. Hell, S. W. \& Wichmann, J. Breaking the diffraction resolution limit by stimulated emission: stimulated-emission-depletion fluorescence microscopy. Opt. Lett. 19, 780-782 (1994).

2. Juette, M. F. et al. Three-dimensional sub-100 $\mathrm{nm}$ resolution fluorescence microscopy of thick samples. Nat. Methods 5, 527-529 (2008).

3. Huang, F. et al. Ultra-high resolution $3 \mathrm{D}$ imaging of whole cells. Cell 166, 10281040 (2016).

4. $\mathrm{Xu}, \mathrm{F}$. et al. Three-dimensional nanoscopy of whole cells and tissues with in situ point spread function retrieval. Nat. Methods 17, 531-540 (2020).

5. de Boer, P., Hoogenboom, J. P. \& Giepmans, B. N. G. Correlated light and electron microscopy: ultrastructure lights up! Nat. Methods 12, 503-513 (2015).

6. Ando, T. et al. The 2018 correlative microscopy techniques roadmap. J. Phys. D Appl. Phys. 51, 443001-446463 (2018).

7. Hoffman, D. P. et al. Correlative three-dimensional super-resolution and block-face electron microscopy of whole vitreously frozen cells. Science 367, eaaz5357 (2020)

8. Udalagama, C. N. B., Bettiol, A. A. \& Watt, F. Stochastic spatial energy deposition profiles for $\mathrm{MeV}$ protons and keV electrons. Phys. Rev. B 80, 224107 (2009).

9. Chen, X. et al. Whole-cell imaging at nanometer resolutions using fast and slow focused helium ions. Biophys. J. 101, 1788-1793 (2011). 
10. Bettiol, A. A., Mi, Z. \& Watt, F. High-resolution fast ion microscopy of single whole biological cells. Appl. Phys. Rev. 3, 041102 (2016).

11. Mi, Z. et al. Subwavelength imaging through ion-beam-induced upconversion. Nat. Commun. 6, 8832 (2015).

12. Boden, S. A., Franklin, T. M. W., Scipioni, L., Bagnall, D. M. \& Rutt, H. N. Ionoluminescence in the helium ion microscope. Microsc. Microanal. 18, 1253 1262 (2012).

13. Doherty, M. W. et al. The nitrogen-vacancy colour centre in diamond. Phys. Rep. 528, 1-45 (2013).

14. Chang, Y.-R. et al. Mass production and dynamic imaging of fluorescent nanodiamonds. Nat. Nanotechnol. 3, 284-288 (2008).

15. Mochalin, V. N., Shenderova, O., Ho, D. \& Gogotsi, Y. The properties and applications of nanodiamonds. Nat. Nanotechnol. 7, 11-23 (2012).

16. $\mathrm{Wu}, \mathrm{T}$.-J. et al. Tracking the engraftment and regenerative capabilities of transplanted lung stem cells using fluorescent nanodiamonds. Nat. Nanotechnol. 8, 682-689 (2013).

17. Haziza, S. et al. Fluorescent nanodiamond tracking reveals intraneuronal transport abnormalities induced by brain-disease-related genetic risk factors. Nat. Nanotechnol. 12, 322-328 (2017).

18. McGuinness, L. P. et al. Quantum measurement and orientation tracking of fluorescent nanodiamonds inside living cells. Nat. Nanotechnol. 6, 358-363 (2011).

19. Le Sage, D. et al. Optical magnetic imaging of living cells. Nature 496, 486-489 (2013).

20. Kucsko, G. et al. Nanometre-scale thermometry in a living cell. Nature 500, 54 58 (2013).

21. Glenn, D. R. et al. Single-cell magnetic imaging using a quantum diamond microscope. Nat. Methods 12, 736-738 (2015)

22. Chipaux, M. et al. Nanodiamonds and their applications in cells. Small 14, 1704263 (2018)

23. Carter, S. D. et al. Distinguishing signal from autofluorescence in cryogenic correlated light and electron microscopy of mammalian cells. J. Struct. Biol. 201, 15-25 (2018)

24. Beha, K., Batalov, A., Manson, N. B., Bratschitsch, R. \& Leitenstorfer, A Optimum photoluminescence excitation and recharging cycle of single nitrogen-vacancy centers in ultrapure diamond. Phys. Rev. Lett. 109, 097404 (2012).

25. Siyushev, P. et al. Photoelectrical imaging and coherent spin-state readout of single nitrogen-vacancy centers in diamond. Science 363, 728-731 (2019).

26. Grotz, B. et al. Charge state manipulation of qubits in diamond. Nat. Commun. 3, 729 (2012).

27. Mizuochi, N. et al. Electrically driven single-photon source at room temperature in diamond. Nat. Photon. 6, 299-303 (2012).

28. Tizei, L. H. G. \& Kociak, M. Spatially resolved quantum nano-optics of single photons using an electron microscope. Phys. Rev. Lett. 110, 153604 (2013).

29. Zhang, H., Glenn, D. R., Schalek, R., Lichtman, J. W. \& Walsworth, R. L. Efficiency of cathodoluminescence emission by nitrogen-vacancy color centers in nanodiamonds. Small 13, 1700543 (2017).

30. Lu, H.-C. et al. Far-UV-excited luminescence of nitrogen-vacancy centers Evidence for diamonds in space. Angew. Chem. Int. Ed. 56, 14469-14473 (2017).

31. Grall, R. et al. Impairing the radioresistance of cancer cells by hydrogenated nanodiamonds. Biomaterials 61, 290-298 (2015).

32. Durante, M., Orecchia, R. \& Loeffler, J. S. Charged-particle therapy in cancer: clinical uses and future perspectives. Nat. Rev. Clin. Oncol. 14, 483-495 (2017).

33. D'Autréaux, B. \& Toledano, M. B. ROS as signalling molecules: mechanisms that generate specificity in ROS homeostasis. Nat. Rev. Mol. Cell Biol. 8, 813824 (2007)

34. Polf, J. C. et al. Enhanced relative biological effectiveness of proton radiotherapy in tumor cells with internalized gold nanoparticles. Appl. Phys. Lett. 98, 193702-193702 (2011).

35. $\mathrm{Li}$, S. et al. LET-dependent radiosensitization effects of gold nanoparticles for proton irradiation. Nanotechnology 27, 455101 (2016).

36. Ghita, M. et al. A mechanistic study of gold nanoparticle radiosensitisation using targeted microbeam irradiation. Sci. Rep. 7, 44752 (2017).

37. Štefančíková, L. et al. Effect of gadolinium-based nanoparticles on nuclear DNA damage and repair in glioblastoma tumor cells. J. Nanobiotechnol. 14, 63 (2016)

38. Baumann, M. et al. Radiation oncology in the era of precision medicine. Nat. Rev. Cancer 16, 234-249 (2016).

39. McKay, P. F. et al. Self-amplifying RNA SARS-CoV-2 lipid nanoparticle vaccine candidate induces high neutralizing antibody titers in mice. Nat. Commun. 11, 3523 (2020).

40. Shin, M. D. et al. COVID-19 vaccine development and a potential nanomaterial path forward. Nat. Nanotechnol. (2020).

41. Vanga, S. K. et al. Development of a new light collection and detection system optimized for ion beam induced fluorescence microscopy. Nucl. Instrum. Methods Phys. Res. B 348, 111-114 (2015).

42. Bettiol, A. A., Udalagama, C. N. B. \& Watt, F. A new data acquisition and imaging system for nuclear microscopy based on a field programmable gate array card. Nucl. Instrum. Methods Phys. Res. B 267, 2069-2072 (2009).

43. Yang, C. et al. Textured V-pit green light emitting diode as a wavelengthselective photodetector for fast phosphor-based white light modulation. ACS Photonics 4, 443-448 (2017).

44. Chen, Q. et al. All-inorganic perovskite nanocrystal scintillators. Nature 561, 88-93 (2018)

45. Incerti, S. et al. Geant4-DNA example applications for track structure simulations in liquid water: A report from the Geant4-DNA Project. Med. Phys 45, e722-e739 (2018)

\section{Acknowledgements}

The work was supported by a National Research Foundation Singapore-Competitive Research Programme (NRF-CRP17-2017-05). We are grateful to Qiushui Chen and Xiaogang Liu for providing samples of perovskite quantum dots.

\section{Author contributions}

F.W. and A.A.B. supervised the project. Z.M. and A.A.B. conceived and designed the experiments. Z.M. carried out the ion beam imaging and spectral measurements. H.Q.T. developed the method for nanodiamond counting. C.C. prepared the cells. Z.M. and C.C. performed confocal imaging experiments. Y.D. performed Geant4-DNA simulations. Z.M., C.Y. and S.P.T. constructed the time-resolved ionoluminescence setup and performed lifetime measurements. Z.M. analyzed the data and prepared the figures. Z.M., F.W. and A.A.B. wrote the manuscript with input from the other authors.

\section{Competing interests}

The authors declare no competing interests.

\section{Additional information}

Supplementary information is available for this paper.

Correspondence and requests for materials should be addressed to F.W. and A.A.B 


\section{Figures}
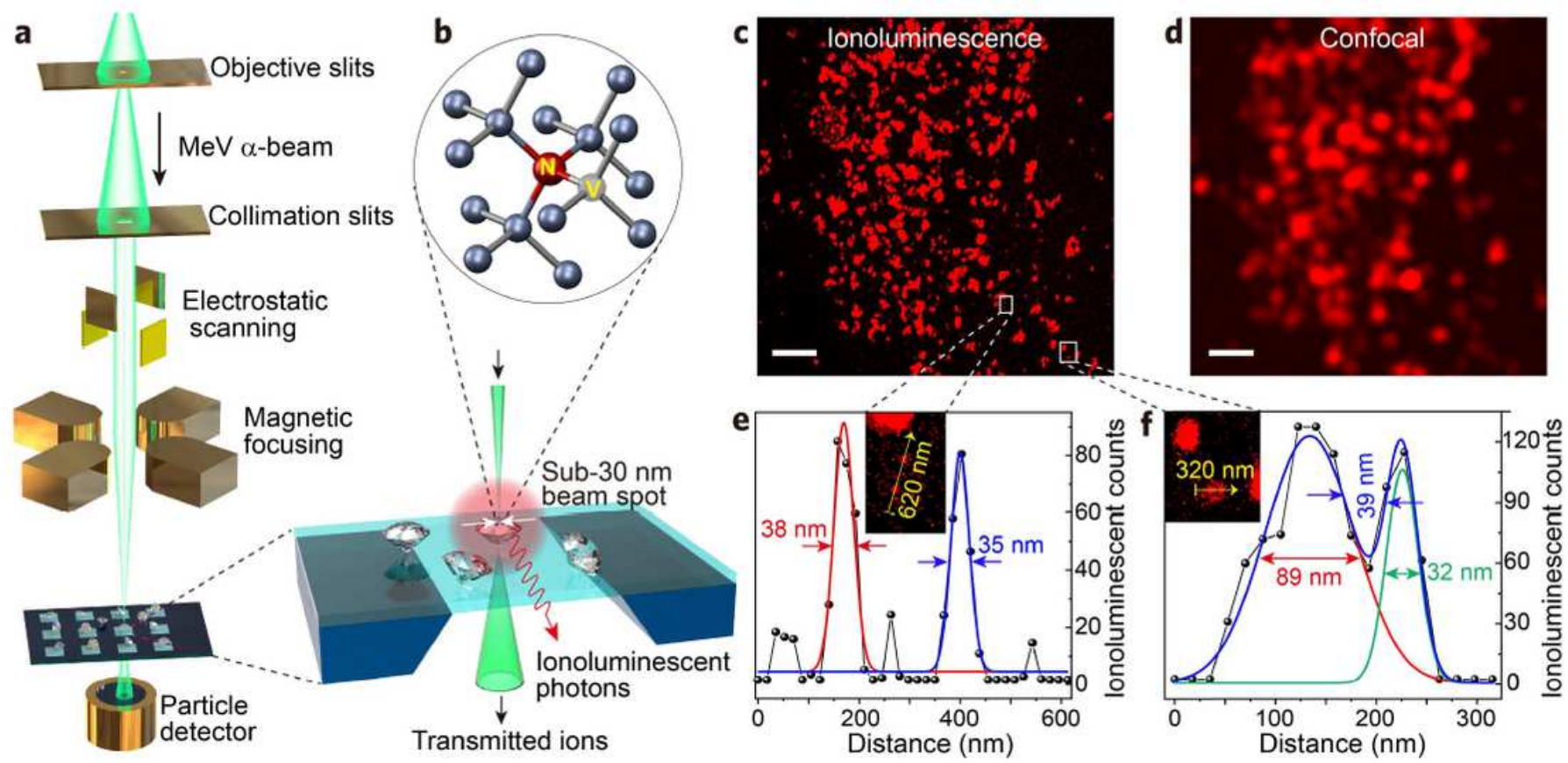

\section{Figure 1}

Ionoluminescence nanoscopy. a, Schematic illustration of the basic beam optics and experimental setup. Note that ionoluminescent photons emitted from the nanodiamonds induced by the a-beam are collected with a parabolic-mirror based system and detected with a photomultiplier tube for ionoluminescence mapping (see Supplementary Fig. 3). b, Nitrogen-vacancy (NV) center in the diamond crystalline structure. c, lonoluminescence image of the nanodiamonds through a-particle excitation. Scale bar, $2 \mu \mathrm{m}$. $d$, Confocal image of the same region of the nanodiamond sample taken by using $543-\mathrm{nm}$ laser excitation. Scale bar, $2 \mu \mathrm{m}$. e, Cross-sectional line profile extracted along the arrow shown in the inset of a highmagnification image that corresponds to the region of interest marked in c, demonstrating the discrimination of two single nanodiamonds with a size of $38 \mathrm{~nm}$ and $35 \mathrm{~nm}$, respectively. $\mathrm{f}$, Crosssectional line profile extracted along the arrow depicted in the inset of a high-magnification image that hosts two single nanodiamonds with a separation distance approximating the resolving limit, directly indicating a sub-40 $\mathrm{nm}$ resolution of the ionoluminescence image. 

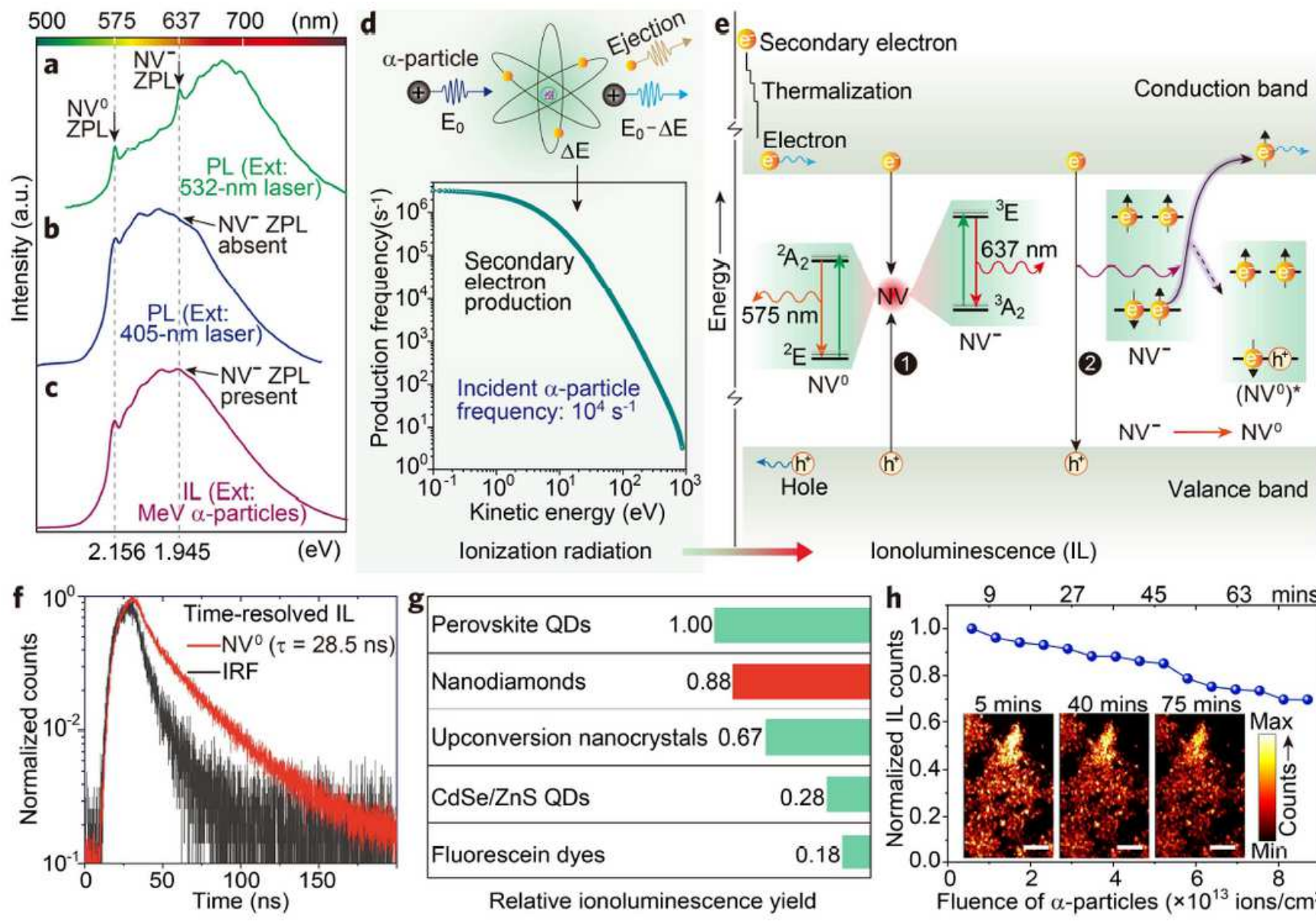

Ionoluminescence (IL)

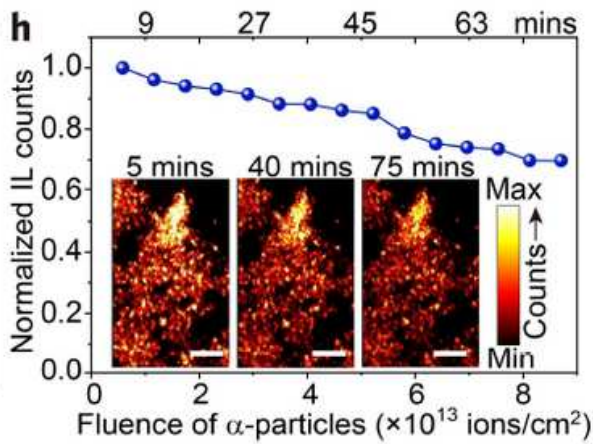

\section{Figure 2}

Mechanistic investigation and characterization of ionoluminescence in nanodiamonds. a, Photoluminescence $(\mathrm{PL})$ spectrum of nanodiamonds excited with a 532-nm laser. b, Photoluminescence spectrum of nanodiamonds excited with a 405-nm laser. c, lonoluminescence (IL) spectrum of nanodiamonds excited with a beam of $1.6 \mathrm{MeV}$ a-particles. d, Illustration of a-particle-induced atomic ionization in producing secondary electrons (top), through energy deposition $(\Delta \mathrm{E})$ of the bombarding aparticles (energy of E0), and calculated energy distribution of the secondary electrons (bottom) in a nanodiamond. e, Proposed mechanism of ionoluminescence through a-particle excitation. Process 1 represents NV-defect-assisted recombination which results in the emission of NVO and $\mathrm{NV}^{-}$. Process 2 represents interband recombination which results in the conversion of $\mathrm{NV}^{-}$to $\mathrm{NV} 0$ through ground-state ionization of $\mathrm{NV}^{-}$, forming $\mathrm{NVO}$ in its excited state of $(\mathrm{NVO})^{\star}$. $\mathrm{f}$, Time-resolved ionoluminescence measurement. Note that the instrumental response function (IRF) was determined by measuring the ionoluminescence response of a fast-decay material (Supplementary Fig. 5 and Note 3). g, Relative ionoluminescence yield measurement. Note that the ionoluminescence yields of nanodiamonds, upconversion nanocrystals (NaYF4: Yb/Tm), CdSe/ZnS quantum dots (QDs), and fluorescein dyes (FITC1907) were normalized with a perovskite-QDs scintillator (Supplementary Figs. 6, 7 and Note 4). h, lonoluminescence intensity profile as a function of the accumulated fluence of $₫$-particles, showing a considerable iono-bleaching resistance of the nanodiamonds. The inserted images, taken at different 
time intervals $(5,40$, and 75 mins), indicate that the emission brightness of the nanodiamonds remains essentially unaltered over time. Scale bars are $2 \mu \mathrm{m}$.
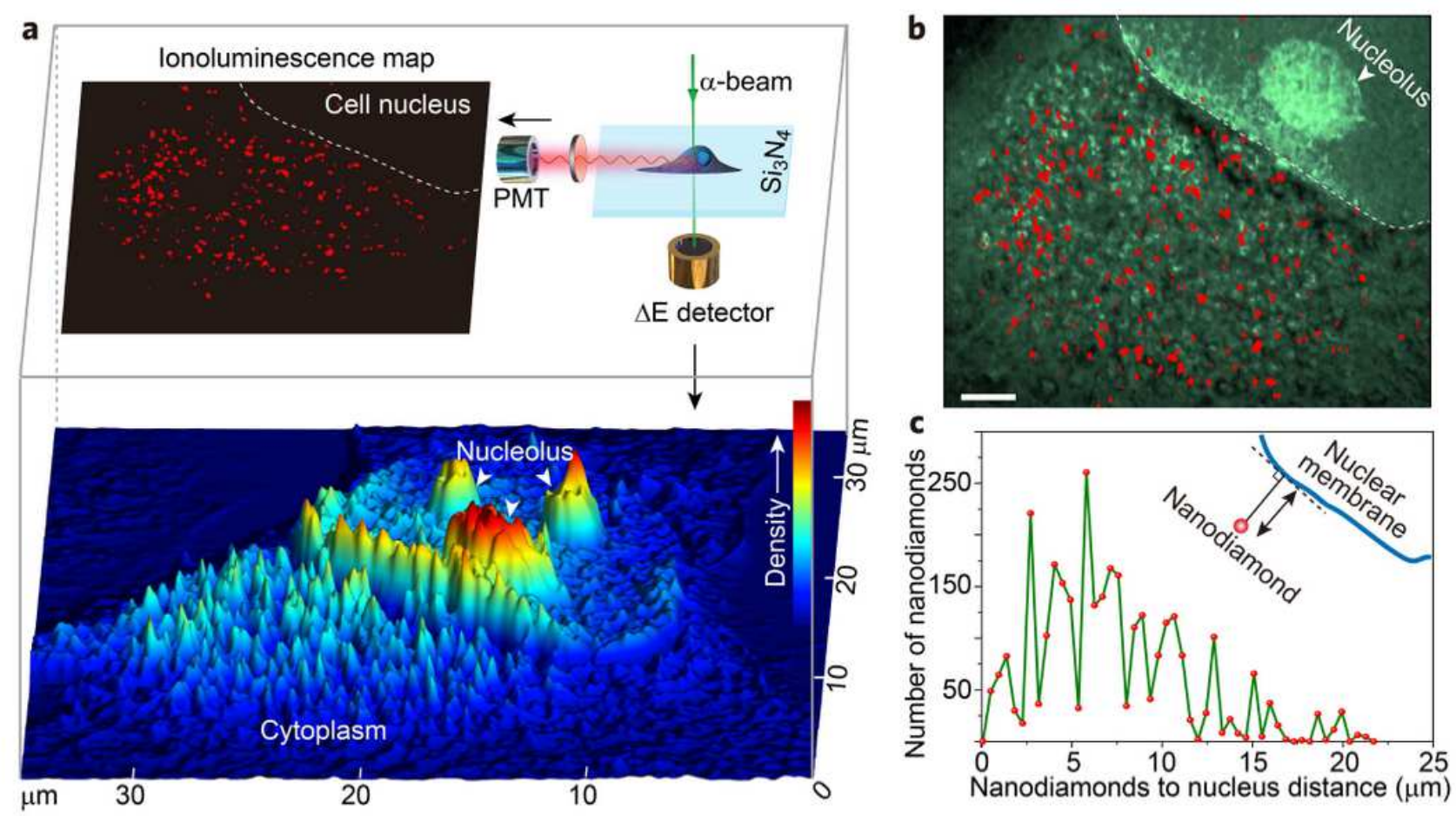

\section{Figure 3}

Correlative ultrastructure and ionoluminescence mapping towards quantitative localization of single nanodiamonds in a whole HeLa cell. a, Demonstration of correlative ultrastructure and ionoluminescence imaging of the HeLa cell with endocytosed nanodiamonds, realized in a single imaging experiment. The basic experimental design (top right) enables simultaneous acquisition of luminescence image of the nanodiamonds and 3D rendering of cell ultrastructure, by capturing a-particle-induced photons with a photomultiplier tube (PMT) and by detecting the energy-loss $(\Delta \mathrm{E})$ of the transmitted a-particles through a Si surface barrier detector, respectively. b, Overlay of the structural and luminescent images presented in a, showing localization of nanodiamonds in the cell. Scale bar, $2 \mu \mathrm{m}$. c, Measurement of the number of intracellular nanodiamonds displayed in $\mathrm{b}$ in terms of their distance to the cell nuclear boundary. 

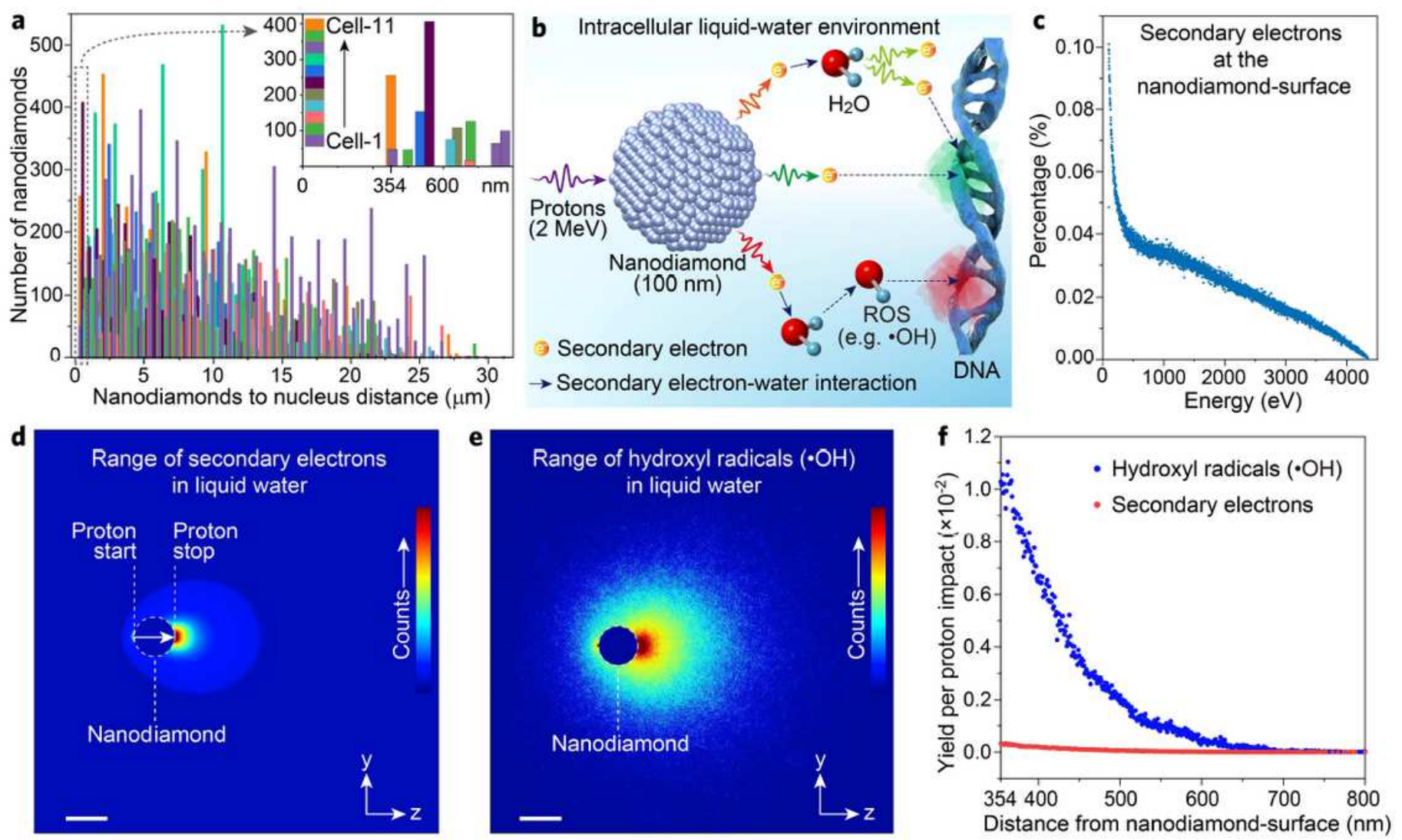

\section{Figure 4}

Evaluation of nanodiamonds as proton radiosensitizers. a, Histogram showing the distribution of nanodiamond-to-nucleus distance that was experimentally measured within $11 \mathrm{HeLa}$ cells. The magnified inset indicates that zero nanoparticles were found within $354 \mathrm{~nm}$ of the nuclear boundary. b, Plausible mechanisms of nanodiamond-mediated proton radiosensitization in damaging nuclear DNA. Secondary electrons can be induced by the 2-MeV protons when they penetrate a nanodiamond. The secondary electrons that emanate from the nanodiamond, as well as subsequent generations of induced electrons, can either damage nuclear DNA directly, or ionize intracellular water molecules in producing reactive oxygen species (ROS) to react with nuclear DNA indirectly. c, Calculated energy distribution of the secondary electrons escaping the nanodiamond at its surface, simulated with Geant4-DNA. d, Image showing range distributions of the secondary electrons in liquid water, simulated with Geant4-DNA. Note that we assumed the protons travel only within the nanodiamond (100 nm diameter). Scale bar, $100 \mathrm{~nm}$. e, Image showing range distributions of hydroxyl radicals $(\cdot \mathrm{OH})$ in liquid water, simulated with Geant4DNA. Scale bar, $100 \mathrm{~nm}$. f, Measurement of the number of hydroxyl radicals and secondary electrons per proton impact as a function of their distances from the nanodiamond-surface. Note that only those traveling more than $354 \mathrm{~nm}$ (the minimum nanodiamond-to-nucleus distance determined in a) were taken into account.

\section{Supplementary Files}


This is a list of supplementary files associated with this preprint. Click to download.

- Supplementarylnformation.pdf 